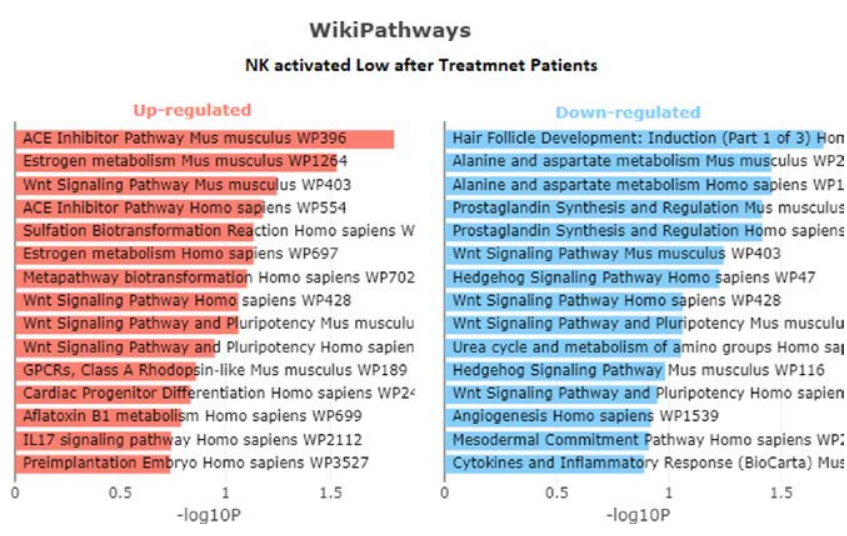

Figure 2. Top wikiPathways enriched in the patients with decreased percentage of synovial infiltrating activated NK after 6 months of tDMARD therapy compared to those who showed increased or unchanged percentage.

Conclusion: Synovial tissue NK cells, resting mast cells, plasma cells and M1 macrophages play major role in response to tDMARD. Genes related to WNT signaling, estrogen metabolism and IL17 signaling can help stratification of patients for a more effective personalized medicine in RA.

References:

[1] Smolen, J.S., D. Aletaha, and I.B. McInnes, Rheumatoid arthritis. Lancet (London, England), 2016. 388(10055): p. 2023-2038.

[2] Safari, F., et al., CRISPR and personalized Treg therapy: new insights into the treatment of rheumatoid arthritis. Immunopharmacology and immunotoxicology, 2018. 40(3): p. 201-211.

[3] Walsh, A.M., et al., Triple DMARD treatment in early rheumatoid arthritis modulates synovial $T$ cell activation and plasmablast/plasma cell differentiation pathways. PloS one, 2017. 12(9): p. e0183928-e0183928.

Disclosure of Interests: None declared

DOI: 10.1136/annrheumdis-2020-eular.6291

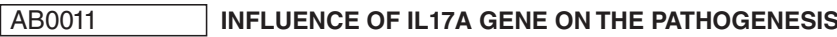 OF IMMUNOGLOBULIN-A VASCULITIS}

F. Genre ${ }^{1}$, S. Remuzgo Martinez ${ }^{1}$, V. Pulito-Cueto ${ }^{1}$, D. Prieto-Peña ${ }^{1}$, B. AtienzaMateo $^{1}$, B. Sevilla ${ }^{2}$, J. Llorca ${ }^{3}$, N. Ortego ${ }^{4}$, L. Lera-Gómez ${ }^{1}$, M. Leonardo ${ }^{5}$, A. Peñalba ${ }^{5}$, M. J. Cabero ${ }^{5}$, L. Martín-Penagos ${ }^{6}$, J. A. Miranda-Filloy ${ }^{7}$, A. Navas Parejo $^{8}$, D. De Argila ${ }^{9}$, M. Aragües ${ }^{9}$, E. Rubio-Romero ${ }^{10}$, M. Leon Luque ${ }^{10}$, J. M. Blanco-Madrigal ${ }^{11}$, E. Galindez ${ }^{11}$, J. Martin Ibanez ${ }^{12}$, S. Castañeda ${ }^{13}$, R. Blanco ${ }^{1}$, M. A. González-Gay 1,14,15, R. López-Mejías ${ }^{1} .{ }^{1}$ Research Group on Genetic Epidemiology and Atherosclerosis in Systemic Diseases and in Metabolic Bone Diseases of the Musculoskeletal System, IDIVAL, Hospital Universitario Marqués de Valdecilla, Santander, Spain; ${ }^{2}$ Division of Pediatrics, Hospital Universitario San Cecilio, Granada, Spain; ${ }^{3}$ Epidemiology and Computational Biology Department, Universidad de Cantabria, and CIBERESP, IDIVAL,, Santander, Spain; ${ }^{4}$ Systemic Autoimmune Diseases Unit, Hospital Universitario San Cecilio, Granada, Spain; ${ }^{5}$ Division of Pediatrics, Hospital Universitario Marqués de Valdecilla, Santander, Spain; ${ }^{6}$ Nephrology Department, Hospital Universitario Marqués de Valdecilla, IDIVAL-REDINREN, Santander, Spain; ${ }^{7}$ Division of Rheumatology, Hospital Universitario Lucus Augusti, Lugo, Spain; ${ }^{8}$ Nephrology Department, Hospital Universitario San Cecilio, Granada, Spain; ${ }^{9}$ Dermatology Department, Hospital Universitario de La Princesa, Madrid, Spain; ${ }^{10}$ Rheumatology Department, Hospital Universitario Virgen del Rocío, Sevilla, Spain; ${ }^{11}$ Rheumatology Department, Hospital Universitario de Basurto, Bilbao, Spain; ${ }^{12}$ Instituto de Parasitología y Biomedicina 'López-Neyra', CSIC, PTS Granada, Granada, Spain;

${ }^{13}$ Rheumatology Department, Hospital Universitario de La Princesa, Madrid, Spain; 14 School of Medicine, University of Cantabria, Santander, Spain; 15 Cardiovascular Pathophysiology and Genomics Research Unit, School of Physiology, Faculty of Health Sciences, University of the Witwatersrand, Santander, Spain

Background: Cytokines signaling pathway genes represent a key component of the genetic network implicated in the pathogenesis of Immunoglobulin-A vasculitis (IgAV) [1], an inflammatory vascular pathology. Interleukin (IL)17A is a genetic risk locus for autoimmune diseases, such as giant cell arteritis [2] and spondyloarthritis [3].

Objectives: To determine the potential influence of IL17A on IgAV.

Methods: Five IL17A tag polymorphisms (rs4711998, rs8193036, rs3819024, rs2275913 and rs7747909) were genotyped in 360 Caucasian patients with IgAV and 1,003 sex and ethnically matched healthy controls.
Results: No statistically significant differences between patients with IgAV and healthy controls were observed when each IL17A genetic variant was analyzed independently. Similarly, no statistically significant differences between patients with IgAV and healthy controls were found when the five IL17A polymorphisms were evaluated combined conforming haplotypes. In addition, there were no statistically significant differences in genotype, allele and haplotype frequencies of IL17A when patients with IgAV were stratified according to the age at disease onset or to the presence/absence of gastrointestinal or renal manifestations. Conclusion: Our results do not support an influence of $I L 17 \mathrm{~A}$ on the pathogenesis of IgAV.

References:

[1] Autoimmun Rev 2018; 17: 301-15

[2] Ann Rheum Dis 2014; 73: 1742-5

[3] Mediators Inflamm 2018; 2018: 1395823.

Acknowledgments: This study was supported by European Union FEDER funds and "Fondo de Investigaciones Sanitarias" (grant PI18/00042) from 'Instituto de Salud Carlos III' (ISCIII, Health Ministry, Spain). RL-M is a recipient of a Miguel Servet type I programme fellowship from the ISCIII, co-funded by the European Social Fund (ESF, 'Investing in your future') (grant CP16/00033) SR-M is supported by funds of the RETICS Program (RD16/0012/0009) (ISCIII, co-funded by the European Regional Development Fund (ERDF)). VP-C is supported by a pre-doctoral grant from IDIVAL (PREVAL 18/01). LL-G is supported by funds of PI18/00042 (ISCIII, co-funded by ERDF).

Disclosure of Interests: Fernanda Genre: None declared, Sara Remuzgo Martinez: None declared, Verónica Pulito-Cueto: None declared, D. Prieto-Peña: None declared, Belén Atienza-Mateo: None declared, Belén Sevilla: None declared, Javier Llorca: None declared, Norberto Ortego: None declared, Leticia Lera-Gómez: None declared, Maite Leonardo: None declared, Ana Peñalba: None declared, María Jesús Cabero: None declared, Luis Martín-Penagos: None declared, Jose Alberto Miranda-Filloy: None declared, Antonio Navas Parejo: None declared, Diego de Argila: None declared, Maximiliano Aragües: None declared, Esteban Rubio-Romero: None declared, MANUEL LEON LUQUE: None declared, Juan María Blanco-Madrigal: None declared, E. Galindez: None declared, Javier Martin Ibanez: None declared, Santos Castañeda: None declared, Ricardo Blanco Grant/research support from: Abbvie, MSD and Roche, Consultant of: Abbvie, Pfizer, Roche, Bristol-Myers, Janssen and MSD, Speakers bureau: Abbvie, Pfizer, Roche, Bristol-Myers, Janssen, Lilly and MSD, Miguel A González-Gay Grant/research support from: Pfizer, Abbvie, MSD, Speakers bureau: Pfizer, Abbvie, MSD, Raquel López-Mejías: None declared

DOI: 10.1136/annrheumdis-2020-eular.641

\section{AB0012 ROLE OF IRF5 GENE ON THE PATHOGENESIS OF IMMUNOGLOBULIN-A VASCULITIS}

S. Remuzgo Martinez ${ }^{1}$, F. Genre ${ }^{1}$, V. Pulito-Cueto ${ }^{1}$, D. Prieto-Peña ${ }^{1}$, B. AtienzaMateo $^{1}$, B. Sevilla ${ }^{2}$, J. Llorca ${ }^{3}$, N. Ortego ${ }^{4}$, L. Lera-Gómez ${ }^{1}$, M. Leonardo $^{5}$, A. Peñalba ${ }^{5}$, M. J. Cabero ${ }^{5}$, L. Martín-Penagos ${ }^{6}$, J. A. Miranda-Filloy ${ }^{7}$, A. Navas Parejo $^{8}$, J. Sanchez Perez ${ }^{9}$, M. Aragües ${ }^{9}$, E. Rubio ${ }^{10}$, M. Leon Luque ${ }^{10}$, J. M. Blanco-Madrigal ${ }^{11}, E$. Galinde ${ }^{11}$, J. Martin Ibanez ${ }^{12}$, S. Castañeda ${ }^{13}$, R. Blanco ${ }^{1}$, M. A. González-Gay 1,14,15, R. López-Mejías ${ }^{1} .{ }^{1}$ Research Group on Genetic Epidemiology and Atherosclerosis in Systemic Diseases and in Metabolic Bone Diseases of the Musculoskeletal System, IDIVAL, Hospital Universitario Marqués de Valdecilla, Santander, Spain; ${ }^{2}$ Division of Pediatrics, Hospital Universitario San Cecilio, Granada, Granada, Spain; ${ }^{3}$ Epidemiology and Computational Biology Department, Universidad de Cantabria, and CIBERESP, IDIVAL, Santander, Spain; ${ }^{4}$ Systemic Autoimmune Diseases Unit, Hospital Universitario San Cecilio, Granada, Spain; ${ }^{5}$ Division of Pediatrics, Hospital Universitario Marqués de Valdecilla, Santander, Spain; ${ }^{6}$ Nephrology Department, Hospital Universitario Marqués de Valdecilla, IDIVAL-REDINREN, Santander, Spain; ${ }^{7}$ Division of Rheumatology, Hospital Universitario Lucus Augusti, Lugo, Spain; ${ }^{8}$ Nephrology Department, Hospital Universitario San Cecilio, Granada, Spain; ${ }^{9}$ Dermatology Department, Hospital Universitario de La Princesa, Madrid, Spain; ${ }^{10}$ Rheumatology Department, Hospital Universitario Virgen del Rocío, Sevilla, Spain; ${ }^{11}$ Rheumatology Department, Hospital Universitario de Basurto, Bilbao, Spain; ${ }^{12}$ Instituto de Parasitología y Biomedicina 'López-Neyra', CSIC, PTS Granada, Granada, Spain;

${ }^{13}$ Rheumatology Department, Hospital Universitario de La Princesa,, Madrid, Spain; 14 School of Medicine, University of Cantabria, Santander, Spain; 15 Cardiovascular Pathophysiology and Genomics Research Unit, School of Physiology, Faculty of Health Sciences, University of the Witwatersrand, Johannesburg, South Africa

Background: Interferon signaling pathway plays a relevant role in autoimmunity. Genetic variants in the interferon regulatory factor (IRF) 5 gene, that encodes the major regulator of the type I interferon induction [1], have been related to the development of several inflammatory diseases [2]. 
Objectives: To determine the influence of IRF5 on Immunoglobulin-A vasculitis (IgAV), an inflammatory vascular disease.

Methods: Three IRF5 polymorphisms (rs2004640, rs2070197 and rs10954213) representative of 3 different haplotype blocks were genotyped in 372 Caucasian patients with IgAV and 876 sex and ethnically matched healthy controls.

Results: No statistically significant differences between patients with IgAV and controls were observed when each IRF5 polymorphism was analyzed independently. Similarly, no statistically significant differences between patients with IgAV and controls were found when IRF5 polymorphisms were evaluated combined conforming haplotypes. Additionally, there were no statistically significant differences in genotype, allele and haplotype frequencies of IRF5 when patients with IgAV were stratified according to the age at disease onset or to the presence/absence of gastrointestinal or renal manifestations.

Conclusion: Our results do not support an influence of IRF5 on the pathogenesis of IgAV.

References:

[1] Nat Immunol 2011; 12: 231-8;

[2] Arthritis Res Ther 2014; 16: R146.

Acknowledgments: This study was supported by European Union FEDER funds and "Fondo de Investigaciones Sanitarias" (grant PI18/00042) from 'Instituto de Salud Carlos III' (ISCIII, Health Ministry, Spain). RL-M is a recipient of a Miguel Servet type I programme fellowship from the ISCIII, co-funded by the European Social Fund (ESF, 'Investing in your future') (grant CP16/00033). SR-M is supported by funds of the RETICS Program (RD16/0012/0009) (ISCIII, co-funded by the European Regional Development Fund (ERDF)). VP-C is supported by a pre-doctoral grant from IDIVAL (PREVAL 18/01). LL-G is supported by funds of PI18/00042 (ISCIII, co-funded by ERDF).

Disclosure of Interests: Sara Remuzgo Martinez: None declared, Fernanda Genre: None declared, Verónica Pulito-Cueto: None declared, D. Prieto-Peña: None declared, Belén Atienza-Mateo: None declared, Belén Sevilla: None declared, Javier Llorca: None declared, Norberto Ortego: None declared, Leticia Lera-Gómez: None declared, Maite Leonardo: None declared, Ana Peñalba: None declared, María Jesús Cabero: None declared, Luis Martín-Penagos: None declared, Jose Alberto Miranda-Filloy: None declared, Antonio Navas Parejo: None declared, Javier Sanchez Perez: None declared, Maximiliano Aragües: None declared, Esteban Rubio: None declared, MANUEL LEON LUQUE: None declared, Juan María Blanco-Madrigal: None declared, E. Galindez: None declared, Javier Martin Ibanez: None declared, Santos Castañeda: None declared, Ricardo Blanco Grant/research support from: Abbvie, MSD and Roche, Consultant of: Abbvie, Pfizer, Roche, Bristol-Myers, Janssen and MSD, Speakers bureau: Abbvie, Pfizer, Roche, Bristol-Myers, Janssen, Lilly and MSD, Miguel A González-Gay Grant/research support from: Pfizer, Abbvie, MSD, Speakers bureau: Pfizer, Abbvie, MSD, Raquel López-Mejías: None declared

DOI: 10.1136/annrheumdis-2020-eular.1023

\section{AB0013 HLA ASSOCIATION WITH SYSTEMIC SCLEROSIS (SSC) IN NORTH INDIAN POPULATION AND FAMILIAL INHERITANCE PATTERNS}

S. Machhua ${ }^{1}$, R. Minz ${ }^{2}$, S. K. Sharma ${ }^{3}$, H. Singh ${ }^{2}$, Y. Kumar ${ }^{2}$, S. Anand ${ }^{4}$, S. Handa ${ }^{5}$, S. Singh ${ }^{6} .{ }^{1}$ Post Graduate Institute of Medical Education \& Research, Chandigarh, Immunopathology, Chandigarh, India; ${ }^{1}$ Post Graduate Institute of Medical Education \& Research, Chandigarh, Immunopathology, Chandigarh, India; ${ }^{3}$ Post Graduate Institute of Medical Education \& Research, Chandigarh, Internal Medicine, Chandigarh, India; ${ }^{1}$ Post Graduate Institute of Medical Education \& Research, Chandigarh, Immunopathology, Chandigarh, India; ${ }^{5}$ Post Graduate Institute of Medical Education \& Research, Chandigarh, Dermatology Venereology Leprology, Chandigarh, India; ${ }^{6}$ Post Graduate Institute of Medical Education \& Research, Chandigarh, Department of Pediatrics, Chandigarh, India

Background: It is widely believed that SSc develops in an individual with a permissive genetic makeup.Genetic influences have long been suspected to impact SSc. In families with a history of SSc, the incidence of disease can range from 1.5 to $1.7 \%$ (1). There are several reports of familial occurrence and certain alleles of the HLA system have been associated with the disease (2).No Indian data pertaining to genetic basis of systemic sclerosis is present. Understanding the genetic basis of the disease will help us in defining the biomarkers of the disease in the population that can help in early diagnosis and prognosis.

Objectives: To study HLA association with Systemic sclerosis (SSc) in North Indian Population and its genetic susceptibility to familial systemic sclerosis.

Methods: A total of 150 SSc patients diagnosed by following ACR and EULAR criteria and 150 control subjects, were genotyped for HLA-A, B, DRB1, DQB1 loci by Luminex® 200 Instrument (USA). The association of alleles with disease susceptibility was tested by Chi-square test and Fisher's exact test.
HLA Typing for HLA class I (A, B, C) and II(DR,DQ,DP) for familial study of systemic sclerosis in 2 families was performed by Next Generation Sequencing(NGS) with illumina MiniSeq using MIA FORA NGS Kits from IMMUCOR Antinuclear patterns (ANA) and specific antibodies were detected by indirect Immunofluorescence and Immunoblot (Euroline, Germany).

Results: Strong disease associations were observed for haplotypes $A^{*} 24(\mathrm{OR}=1.7 ;<\quad 0.02), \quad A^{*} 32(\mathrm{OR}=2.8 ;<\quad 0.02), \quad \mathrm{B}^{*} 35(\mathrm{OR}=1.7 ;<\quad 0.03)$ DRB1*11(OR=2.1; $<0.007)$. The reduced frequencies of haplotypes $A^{*} 68(P<$ $0.05), D R B 1^{*} 10(P<0.05), D R B 1 * 12(P<0.00)$ among patients suggested a protective association. There was no statistical association found with HLA $\mathrm{DQB}^{*} 1$.

Through NGS we observed that in the $1^{\text {st }}$ family haplotypes HLA $-A^{*} 11,32,24$; $\mathrm{B}^{*}$ 51, 55, 35; C*-14, 04; DRB1*15, 04; DQB1*05, 03; DPB1*04, 26 appears in affected family members with serological abnormalities. In the $2^{\text {nd }}$ family both mother and daughter had same set of haplotypes except DQB1 with serological abnormalities. The haplotypes DPB $1{ }^{*} 04$ was present in all the diseased individuals of both the families (Fig. 1 and table 1).

Table 1. NGS HLA typing report

\begin{tabular}{lcccccc}
\hline & A & B & C & DRB1 & DQB1 & DPB1 \\
\hline F1 & 1124 & 3515 & 0404 & 1515 & 0505 & 0226 \\
F2 & 1132 & 5155 & 1404 & 1504 & 0503 & 0404 \\
F3 & 1124 & 3555 & 0404 & 1515 & 0505 & 2604 \\
F4 & 3211 & 5115 & 1404 & 1504 & 0503 & 0204 \\
F5 & 2433 & 3544 & 0407 & 1507 & 0502 & 2614 \\
F6 & 1124 & 3555 & 0404 & 1515 & 0505 & 0426 \\
F7 & 1124 & 3555 & 0404 & 1515 & 0505 & 0426 \\
F8 & 2432 & 5135 & 1404 & 0415 & 0305 & 2604 \\
F9 & 1132 & 5155 & 1404 & 1504 & 0503 & 0404 \\
F10 & 1133 & 4452 & 0712 & 1107 & 0203 & 0413 \\
F11 & 1133 & 4452 & 0712 & 1107 & 0303 & 0413 \\
\hline
\end{tabular}

Fig. 1

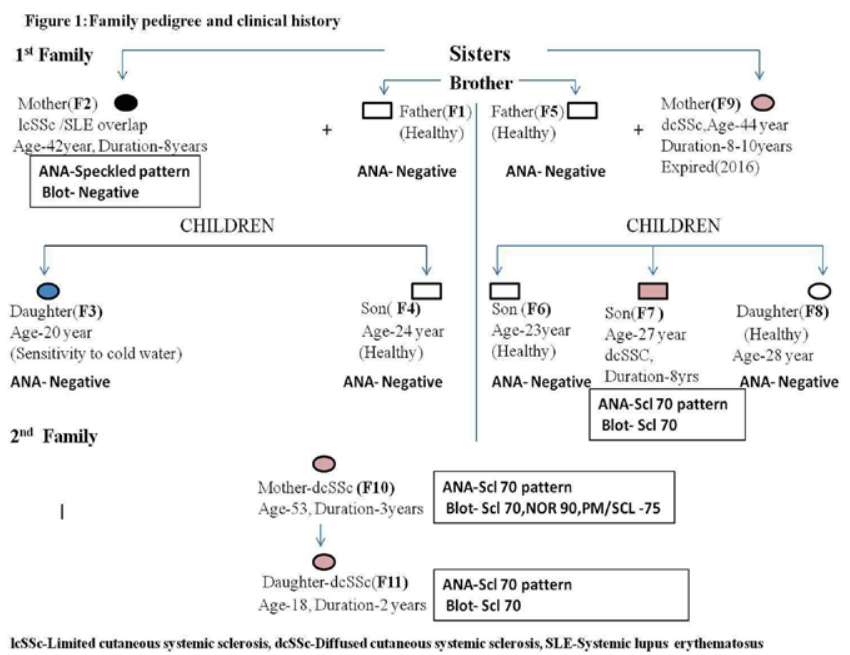

Conclusion: The risk alleles $A^{*} 24,32 ; B^{*} 35$; DRB1 $1^{*} 11$ were found to be associated with North Indian cohort of SSc, while the protecting alleles were $A^{*} 68$; DRB1*10, 12. These risk alleles were present in the SSc affected family members and the protective alleles were absent in the same. Surprisingly, even healthy members carried the same risk alleles but did not manifest the disease or have serological evidence of the same. We have not excluded occurrence of disease at a later age, as presently the healthy siblings are young. Thus our study indicates that though HLA association are found with SSc but many other factors like HLA $\left(H L A{ }^{*} C, D P B 1{ }^{*}\right)$ or non HLA genes as wells as epigenetic factors might also play a role in disease manifestation and severity.

References:

[1] Luo $Y$, Wang $Y$, Wang $Q$, et al. Systemic sclerosis: genetics and epigenetics. J Autoimmun.2013; 41:161-67.

[2] de Juan $\mathrm{MD}^{1}$, Belzunegui J, Belmonte I, Barado J, Figueroa M, Cancio J, Vidal S, Cuadrado E. An immunogenetic study of familial scleroderma. Ann Rheum Dis. 1994 Sep; 53(9):614-7.

Acknowledgments: The technical help of Mr.Manoj Kumar and Mr.Vinkesh are hereby gratefully acknowledged Indian Council of Medical Research(Funding of Fellowship)

Disclosure of Interests: None declared

DOI: 10.1136/annrheumdis-2020-eular.5338 